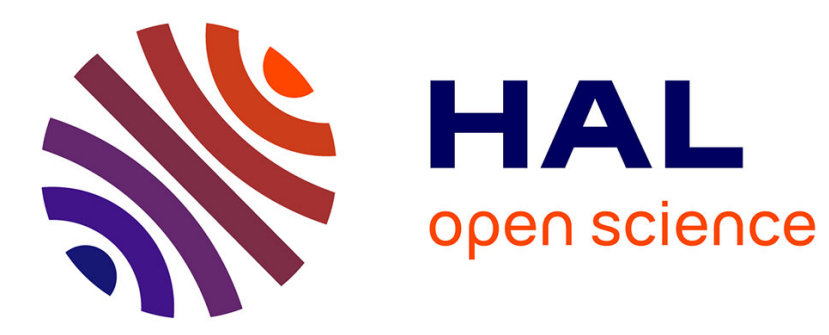

\title{
Structures conceptuelles, représentation des objets et des relations entre les objets.
}

S. Chemlal, Françoise Cordier

\section{To cite this version:}

S. Chemlal, Françoise Cordier. Structures conceptuelles, représentation des objets et des relations entre les objets.. Canadian Journal of Experimental Psychology, 2006, 60 (1), pp.7-23. 10.1037/cjep2006003 . hal-00086727

\section{HAL Id: hal-00086727 https://hal.science/hal-00086727}

Submitted on 19 Jul 2006

HAL is a multi-disciplinary open access archive for the deposit and dissemination of scientific research documents, whether they are published or not. The documents may come from teaching and research institutions in France or abroad, or from public or private research centers.
L'archive ouverte pluridisciplinaire HAL, est destinée au dépôt et à la diffusion de documents scientifiques de niveau recherche, publiés ou non, émanant des établissements d'enseignement et de recherche français ou étrangers, des laboratoires publics ou privés. 


\title{
STRUCTURES CONCEPTUELLES, REPRESENTATION DES OBJETS
}

\section{ET DES RELATIONS ENTRE LES OBJETS}

\author{
Soulaimane Chemlal et Françoise Cordier
}

Laboratoire Langage \& Cognition (LaCo)

FRE CNRS 2725

99 avenue du Recteur Pineau

F - 86000 Poitiers 


\title{
Résumé
}

Cet article présente un panorama général de l'étude psychologique des concepts liés aux objets, l'insistance étant mise sur les conceptualisations les plus récentes et les derniers développements de la recherche dans ce domaine. Ces derniers développements portent essentiellement d'une part sur la notion de contexte et son rôle dans l'organisation des concepts, et d'autre part sur la notion de relations entre traits - et singulièrement les relations de nature causale. Des illustrations expérimentales viennent étayer l'analyse théorique du domaine. Nous terminons par une réflexion sur les recherches ayant pour objet les déficits catégoriels spécifiques, en les positionnant par rapport à l'évolution des théories de la catégorisation.

Mots-clés: concept, contexte, trait, relation de causalité, essentialisme.

\author{
Abstract \\ This article provides an overview of psychological studies of object \\ concepts, highlighting the more recent re-conceptualizations, and the latest \\ developments in research in this field. These developments have tended to focus \\ on the notion of context, as well as on the notion of causal relations between \\ features. Our theoretical analysis of this field is backed up by experimental \\ illustrations. We complete with an examination of " category-specific \\ impairments " studies, in the light of the evolution of concept theories. \\ Key-words: concept, context, feature, causal relation, essentialism.
}


Les connaissances liées aux objets ont été l' enjeu de plusieurs théorisations successives. Dans toute évolution scientifique normale, des phases plus ou moins longues d' accumulation des données sont suivies par des périodes où le nombre de faits à reconsidérer est suffisamment important pour que le besoin d' une nouvelle cohérence s' exprime plus fortement. De nouvelles propositions sont alors avancées, en réaction aux attendus précédents. Le but de cet article est de mettre l' accent sur la chaîne de bouleversements paradigmatiques qui a touché les théories conceptuelles, l' insistance étant mise cependant sur les bouleversements les plus récents.

Les concepts sont des unités élémentaires du traitement cognitif. Le monde réel n' étant donné à l' esprit que tel un flot continu et dynamique de stimulations, c' est à l' individu d' élaborer des représentations susceptibles d' être traitées, stockées et transmises. $C^{\prime}$ est pourquoi on conçoit les représentations conceptuelles comme des intermédiaires entre le monde perçu et le monde représenté ayant, selon la formule de Neisser (1987) " un pied dans la perception et un autre dans la cognition ". Elles posent d' emblée la question de l' interaction esprit / monde (Medin \& Coley, 1998).

La catégorisation est ainsi un processus fondamental de prise d' information sur le monde. "Concevoir" et "énoncer" constituant deux activités cognitives intimement liées, l' étude des concepts se trouve concernée par les recherches sur les processus langagiers, et plus généralement sur les activités cognitives liées à l' utilisation d' informations sémantiques (compréhension et production de textes, résolution de problèmes, mémorisation). Les théories de la catégorisation se 
trouvent donc occuper une position stratégique particulière par leur résonance dans d' autres champs de la cognition.

Nous considérerons quatre grandes familles théoriques qui traitent des représentations des objets, en en respectant globalement la chronologie. Nous commencerons par la théorie classique et les théories de l' air de famille de façon conjointe. Nous les commenterons succinctement car elles sont bien connues dans leurs attendus. Les théories dites " de l' exemplaire " et les théories fondées sur les connaissances viendront ensuite, que nous développerons plus largement. Nous terminerons cet article en recherchant l' évolution des théories conceptuelles au travers des interprétations des déficits catégoriels spécifiques dans le champ de la neuropsychologie.

\section{Théorie classique et théories de l'air de famille}

La question posée est de caractériser l'intension des représentations telle qu'elle rende compte de leur extension, c'est-à-dire d'un regroupement d'objets. Les deux conceptions de la structure conceptuelle que sont la conception classique et la conception de l' air de famille (conception probabiliste)Medin \& Coley, 1998; Medin \& Heit, 1998; Medin \& Wattenmaker, 1987) atteignent ce but dans la mesure où la représentation contient des conditions ou des règles claires d'appartenance, un ensemble circonscrit de traits sémantiques qui constitue la signification. Il n' est pas utile de présenter ces deux conceptions en détail. Elles ont été largement présentées par ailleurs (Cordier, 2002; Komatsu, 1992; Pothos, in press). 
Dans ces conceptions, la signification des objets ne change pas quelle que soit la situation. Ainsi, les concepts sont vus comme une liste de traits indépendants dans un cas ou corrélés dans l'autre, traits communs de manière plus ou moins stricte à tous les exemplaires.

Les traits jouent un double rôle. D’une part, qu'ils soient définitoires ou seulement probables, leur combinaison permet de décrire l'ensemble des exemplaires de la catégorie, l'extension. Ils déterminent l'appartenance catégorielle. Les objets d' une même catégorie partagent des traits en totalité ou en grande partie et entretiennent ainsi une relation de similitude (Heit, 1997; Murphy \& Medin, 1985; Rips, 1989). Le degré de similarité est considéré comme le principe organisateur des catégories et donc de la mémoire sémantique.

Catégoriser implique d' estimer cette similitudeßarsalou, 1991; Hahn \& Chater, 1997; Rips, 1989). On pourrait penser que la notion de similitude est superflue pour la conception classique, puisque les exemplaires d' un même concept sont également semblables entre eux. Cependant, certains auteurs, dans une discussion de résultats expérimentaux obtenus par ailleurs, ont montré que la similitude avec des exemplaires déjà classés affectait même l'application d'une règle simple et explicite (Hahn \& Chater, 1997; Pothos, in press).

De ces considérations générales, on peut noter que les objets qui sont semblables ${ }^{1}$ occupent une place équivalente dans le monde représenté, ont la même signification, engagent les mêmes actions (Neisser, 1987; Reed, 1996; Tijus, Poitrenaud, \& Barcenilla, 1997), et ont souvent la même dénomination. Sur ce dernier point, les travaux de Malt, Sloman, Genneri, Shi et Wang (1999) et

\footnotetext{
${ }^{1}$ Une typologie des propriétés est proposée par Cordier et Tijus (2001). Cette typologie se donne pour but d' unifier le langage employé lorsqu' il s' avère important d' examiner les rôles respectifs des différentes propriétés dans la catégorisation. Deux raisons majeures peuvent être évoquées: une raison théorique, les différentes
} 
Malt, Sloman et Genneri (2003) portent cependant à l' attention les déterminismes propres apportés par le langage. Dans les expériences rapportées, des dessins d' objets dont la fonction est de contenir des liquides, doivent être répartis par des adultes selon leur degré de ressemblance $\left(1^{\circ}\right.$ classement demandé) et selon leur dénomination ( $2^{\circ}$ classement). Les répartitions obtenues en fonction des 2 critères sont grosso modo homogènes, mais certains objets s' écartent de la règle commune. Et ces objets ne sont pas les mêmes selon la langue considérée (anglais, espagnol, chinois). Ces expériences soulignent le caractère multidéterminé du fonctionnement langagier, et ses arrière-plans culturels et sociaux. La dénomination tient ainsi une place particulière comme indicateur des regroupements conceptuels.

Globalement, les théories classique et de l' air de famille se distinguent sur deux points. (1) La conception classique est censée rendre compte de la catégorisation de tout type d' objets alors que la conception de l'air de famille cible la catégorisation des objets du monde réel. Les catégories naturelles contiennent, outre les traits nécessaires et suffisants, les traits présents avec une probabilité notable : ils sont dits caractéristiques. S’il n’y a aucune raison de penser que l'appartenance catégorielle est moins claire ${ }^{2}$, la représentativité des entités diffère puisqu' elles ne possèdent pas toutes le même nombre de traits. (2) La théorie classique tente de cerner une catégorisation logique et idéale alors que la seconde s'attache davantage à décrire la catégorisation naturelle effective. La théorie classique est concernée par les concepts scientifiques et leurs taxonomies. Les

théories conceptualisant les propriétés différemment; une raison méthodologique, les distinctions entre propriétés permettant un grain d' analyse plus fin. 
concepts sont d'ailleurs formalisés comme des fonctions de vérité sur un domaine de connaissance (Frege, cité par Le Ny, 1989). Tiberghien (2003) souligne que de nombreux problèmes d' enseignement des sciences viennent $\mathrm{d}^{\prime}$ une méconnaissance chez l' élève des traits cruciaux, alors que dans le même temps, il utilise des traits caractéristiques (Cordier \& Cordier, 1991; Cordier, Cordier, \& Es Saïdi, 1997). Les travaux de Cordier et Cordier (1991) portent sur la résolution du théorème de Thalès et mettent en évidence que les erreurs des élèves peuvent être trois fois plus nombreuses quand la figure géométrique met en situation des parallèles qui se trouvent situées de part et d' autre d' une intersection, par rapport à une situation où les parallèles se trouvent du même côté de l' intersection. D' autres problèmes sont liés au fait que l' élève se focalise sur des traits définitoires appartenant à d' autres catégories (Chi \&Slotta, 1993) : dans la résolution de problèmes de physique la propriété de Force est catégorisée par l' élève comme une propriété $d^{\prime}$ un objet - la force d' un homme - et non comme un processus.

En résumé, dans ce type d'approche, la notion de similitude est cruciale puisqu'elle organise la structure conceptuelle de deux manières :

- Elle détermine les liens entre les exemplaires d'une catégorie et, au moins pour la conception probabiliste, le degré de typicalité d'un exemplaire.

- Elle détermine la probabilité qu'un nouvel objet soit rangé dans une catégorie donnée.

\footnotetext{
${ }^{2}$ Lorsque l' on demande à des adultes si l' autruche est un oiseau, leur réponse est OUI sans équivoque. Le temps de réaction est cependant plus long. Il est important ici de distinguer deux dimensions : la dimension d' appartenance qui est binaire d' une part; le gradient tepicalité d' autre part.
} 
Les prémices d'un bouleversement paradigmatique : catégorisation en contexte et représentations schématiques

Les approches citées précédemment évoquent l'existence d'une structure conceptuelle responsable de l'élaboration d'une organisation des connaissances en catégories d'objets similaires. C'est la perception de ces objets ainsi que l'analyse de leurs constituants (structuraux) qui vont permettre dinvoquer le concept approprié. Si effectivement la perception de l'objet isolé et de ses caractéristiques perceptives " appelait " le concept, se poserait le problème de la sélection du bon concept parmi les multiples candidats possibles. Les caractéristiques perceptives, comme l'apparence globale, peuvent être ambiguës et évoquer plusieurs concepts différents (de niveau de base notamment). Par exemple, la forme globale du cheval peut appeler les concepts de cheval, de poney.... D'autre part, que se passet-il si un objet n' est pas vu entièrement ? Or la perception des objets rencontrés est rarement optimale, un cheval pouvant être vu de face, de dos, de profil... Et pourtant, les objets sont reconnus dans la plupart des cas, avec un effet de typicalité de la position de face, il est vrai (Palmer, Rosch, \& Chase, 1981).

Le contexte dans lequel l'objet est rencontré peut apporter une aide à la catégorisation. Les objets sont reconnus plus facilement dans un contexte que hors contexte (Kirasic, Siegel, \& Allan, 1980). D' autre part, plusieurs expériences ont illustré la flexibilité des représentations conceptuelles dans des contextes variés : dans une épreuve de compréhension, lorsqu' un même mot (par ex. église) est mentionné dans deux phrases évoquant deux contextes différents (un mendiant qui tend la main versus un village aperçu au loin)et qu' en phase test, l' expérimentateur propose un dessin figurant une propriété évoquée par l' un ou l' autre contexte (un porche ou un clocher), il s' avère que les temps de décision du 
sujet (réponses oui) sont plus courts en situation de congruence contexte - dessin pour le même concept (Denis \& Le Ny, 1986). Ce type d' effet n'est pas pris en compte dans les modèles classique et de l' air de famille, où toute la variabilité liée aux divers contextes est éliminée dans l'abstraction qui aboutit à la catégorie.

Selon Mandler (1979, 1983), catégoriser est une activité cognitive qui ne peut être complètement distinguée des événements au cours desquels elle se produit. Les concepts doivent contenir des informations sur ces événements et leur variabilité. Même la perception du stimulus le plus simple est saturée de connaissances préalables et d'attentes. Nelson (1996) le définit comme une structure d'événement. Le concept est alors relié à une information sur la manière dont les choses se comportent ou sont utilisées, une fonction au sens large.

Les concepts ainsi envisagés sont fortement contextualisés. Ils sont intégrés dans des schémas, structures conceptuelles fondées sur des principes fonctionnels d'organisation. Cette idée n'est pas nouvelle en psychologie et on la trouve déjà chez Bartlett (1932), qui soutenait qu'on se rappelle un matériel nouveau en fonction de structures déjà existantes (cité par Baddeley, 1992; Neisser, 1988). Le concept de schéma a été repris dans les années 70. Désormais, ce concept est évoqué pour caractériser la structure des représentations d'événements statiques, de situations (Bryant, Tversky, \& Franklin, 1992; Mandler, 1979, 1983), d'événements dynamiques, d'actions ( Nelson, 1977; Schank \& Abelson, 1977; cf. Jagot, 2002, pour une recension critique) et d'objets (Le Ny, 1989, 2005; Rumelhart \& Norman, 1981). Dans les schémas, les entités sont liées entre elles parce qu' ensemble, elles concourent à remplir une certaine fonction.

Cette évolution théorique a le mérite de souligner l'existence d'une connaissance conceptuelle des objets fondée non seulement sur une analyse 
interne des propriétés de l'objet mais aussi sur l' analyse des relations externes.

Les structures formées sont plus flexibles, les propriétés à la base de la similitude étant diversifiées et sélectionnées en fonction du contexte. Les théories de

l' exemplaire iront beaucoup plus loin dans cette voie. Cette évolution laisse par contre inchangé le postulat que les processus de formation des concepts -

fondamentalement inductifs - portent sur des représentations symboliques, complexes, sémantiquement interprétables. Ne peut-on en rendre compte par de simples mécanismes associatifs agissant sur des données élémentaires (issues de la perception) fortement connectées, et par une mise en commun de multiples traitements locaux ? Smith (1989) explique des régularités comportementales dans l' apprentissage de concepts chez l' enfant et chez l' adulte en prenant en compte uniquement le traitement de données perceptives, la détection des similitudes entre les objets, sans recourir à l' idée d' un guidage par une représentation abstraite. Bref, en paraphrasant un titre de Brooks (1991), peut-on envisager une catégorisation sans représentation ? Voilà une modification majeure du paradigme initial qu' il faut examiner maintenant.

\section{Théories de l'exemplaire}

Expliquer l' extrême flexibilité de l' utilisation des concepts en fonction du contexte est une des questions considérées par les théories de l' exemplaire. Comment les connaissances abstraites supposées présentes de façon permanente en mémoire à long terme sont-elles acquises et modifiées par l' expérience en sera une seconde. Ces interrogations sont fortement liées aux recherches développées dans le champ de la mémoire, et les modèles de catégorisation basés sur le stockage d' exemplaires se trouvent de fait étroitement articulés avec les études concernant la mémoire 
épisodique. Ces dernières font l' hypothèse de la présence de représentations mnésiques indépendantes (donc stockées dans des registres mnésiques différents) liées d' une part à une information spécifique, épisodique et d' autre part à une information abstraite (Tulving, 1983). Une information particulière peut être retenue pendant fort longtemps (Barsalou, 1990; Brooks, 1978; Tulving, 1972) et l' individu, sensible au contexte de l' apprentissage, y trouve des indices de récupération de l' information. On attribue généralement aux derniers items plus de poids dans l' apprentissage, ce qui permet de rendre compte des effets de récence observés classiquement par ailleurs dans les études sur la mémorisation. Ce courant a accrédité l' idée que l' information liée à un exemplaire (voire à une présentation donnée d' un exemplaire donné) peut être préservée en mémoire. Les recherches expérimentales qui confortent cette hypothèse sont celles qui - par exemple - mettent en évidence la capacité des sujets à se souvenir de la fréquence de présentation d' un item donné dans une liste, de son temps d' exposition, de sa position sérielle, et de façon générale des modalités de la présentation (Lamberts, 1994; Nosofsky, Clark, \& Shin, 1989). Les théories de l' exemplaire souscrivent à l' hypothèse que seul le stockage des informations spécifiques peut rendre compte à la fois des effets contextuels et de la formation d' une catégorie abstraite au moment de la récupération de l' information, par émergence d' un pattern d' activation (de régularités) dans un ensemble d' activités d' arrière-plan. Ces modèles attribuent un rôle important à la familiarité ${ }^{3}$ des différentes sources d' information, qui augmente graduellement durant les processus de traitement, jusqu' à atteindre un seuil suffisant d' activation pour permettre la décision.

Les options sont ainsi radicalement différentes de celles qui constituent le corps des théories précédentes. Alors que, pour ces dernières, une information abstraite de

\footnotetext{
${ }^{3}$ Il existe une forte parenté entre ce concept de familiarité, et celui d' " harmonie "J(oordens \& Becker, 1997).
} 
niveau catégoriel est construite, conservée en mémoire, et récupérable lorsqu' une nouvelle occurrence se présente, l' information en mémoire se résume ici à un vaste ensemble d' exemplaires. Le processus de catégorisation dépend, quant à lui, du degré de similitude de l' instance en cours de traitement avec ces exemplaires stockés, avec lesquels elle se trouve comparée. La décision catégorielle est prise par rapport à l' exemplaire le plus semblable trouvé en mémoire. Dans cette configuration, il n' y a pas nécessité d' avoir recours à une représentation de tendance centrale, permanente en mémoire. Si une information abstraite se trouve stockée - les modèles n' interdisent pas cette option $^{4}$ - elle ne joue pas de rôle particulier au moment de décision : elle est sur le même plan que les autres (Gluck \& Bower, 1988a \& b; Hintzman, 1986; Hintzman \& Ludlam, 1980; Medin \& Schaffer, 1978; Nosofsky, Clark, \& Shin, 1989; Nosofsky, Kruschke, \& McKinley, 1992).

La plupart des recherches expérimentales visant à évaluer ces modèles adoptent une procédure d' apprentissage, utilisant souvent des stimuli peu complexes, variant sur quelques dimensions. Par exemple, Goldstone (1995) apprend aux sujets à discriminer des carrés, variant suivant leur taille et leur brillance. Sloutsky, Lo et Fisher (2001) proposent des visages schématiques, dont la taille du nez et des oreilles varie, pour une tâche d' induction. Dès que le matériel devient plus complexe - comme c' est le cas dans la catégorisation naturelle - il s' avère difficile de mettre en place des procédures qui fassent des prédictions spécifiques et dont les données ne pourraient être expliquées que dans le cadre d' une théorie de l' exemplaire. Les résultats sont assez souvent appelés en renfort par des théories différentes (Estes, 1986; Hintzman \& Ludlam, 1980). Ceci a conduit Malt (1989) à adopter une procédure d' amorçage, pour atteindre les processus de catégorisation on line. Elle utilise un matériel composé de dessins,

\footnotetext{
${ }^{4}$ Par exemple une situation où une personne cherche un prototype d' une catégorie.
} 
certains éléments des dessins étant communs à toute une catégorie, et d' autres propres à un seul dessin (une couleur, par ex.). Sont mises en évidence à la fois une récupération de l' information spécifique, mais aussi une utilisation d' une information catégorielle abstraite. Evidemment, ce second résultat s' insère mal dans les présupposés des modèles de l' exemplaire.

\section{Le concept de similitude}

Une question importante à laquelle tous ces modèles se trouvent confrontés porte sur la théorie de la similitude que l' on se donne pour étalonner la ressemblance des exemplaires et fonder le processus de comparaison. Une trace en mémoire est modélisée comme une liste d' attributs (ou dimensions). Un attribut caractérise ou non un exemplaire donné. Si deux représentations sont caractérisées par la même valeur sur la même dimension, elles ont un attribut commun. Cet attribut est distinctif si les deux représentations ont des valeurs différentes sur la même dimension. Les travaux de Markman et Gentner (1993 a \& b) présentent une modélisation pour laquelle la similitude entre une cible et un exemplaire stocké augmente avec le nombre de leurs propriétés communes et décroît avec leurs différences, et, pour déterminer ces ressemblances et différences, ils proposent un processus " d' alignement des structures ". Cet " alignement " fait une distinction entre des différences reliées à une structure commune (des valeurs différentes sur une dimension donnée : un objet vert et un objet rouge : différences " dans l' alignement ") et des différences non liées à une structure commune (un objet rouge et un objet carré : différences " hors - alignement "), les comparaisons " dans l' alignement " étant celles qui ont le plus de poids dans le traitement cognitif. Ceci répond à une critique qui est souvent faite aux modèles de catégorisation qui se fondent sur la similitude : le nombre des dimensions sur 
lesquelles les items peuvent être comparés étant en principe illimité (une pintade et un cube rouge se ressemblent au sens où ils ont tous deux une masse, par exemple), la similitude y prend la tonalité un peu floue d' un concept insuffisamment contraint pour garder une grande valeur explicative. Si les théories fondées sur l' explication vont s' engouffrer dans cette brèche, ceci va également conduire à des amendements des modèles de l' exemplaire existants. Il semble admis aujourd' hui que les calculs de la similitude sont variables selon la tâche, les consignes données aux sujets, l' ensemble des stimuli (Lamberts, 1994). Les sujets peuvent prendre des options stratégiques, qui seraient les analogues de critères de réponse en détection du signal ou en reconnaissance : la similitude entre les exemplaires est alors modifiable par le jeu d' une attention sélectiveX(Nosofsky et al., 1989). Ces modifications stratégiques, qui reviennent à changer le poids des dimensions, peuvent survenir en cours de tâche, en fonction des informations reçues en retour.

Si la souplesse des processus de catégorisation peut être convenablement prise en compte par les théories de l' exemplaire, il apparaît que la plus grande flexibilité qui résulte du stockage des exemplaires a deux coûts : celui des capacités de stockage requises et/ou celui de l' importance du temps de traitement dans la récupération de l' information, donc un coûtomputationnel. McClelland et Rumelhart (1985) qui s' inscrivent comme les modèled' Hintzman (MINERVA 2) et deNosofsky (ALCOVE) dans le cadre d' un modèle de mémoire distribuée, supposent que les traces des événements se superposent au moment du stockage de l' information (et non pas au moment de la récupération). Cette superposition des traces au moment de l' encodage débouche automatiquement sur une abstraction (tendance centrale), bien que la spécificité des événements puisse être préservée dans une certaine mesure. Mais les traces individuelles ne sont pas gardées séparément en mémoire. La proposition de 
McClelland et Rumelhart n' est pas intégrée dans les modèles de l' exemplaire en tant que telle, dans la mesure où cette conception accepte à la fois la représentation d' une abstraction, et, dans une certaine mesure, celle d' événements spécifiques. Les données de Malt (1989) évoquées plus haut peuvent être interprétées dans ce cadre plus aisément.

Il existe plusieurs sortes de modèles mixtes de la catégorisation (Komatsu, 1992) qui tiennent compte des informations liées à l' exemplaire, mais n' écartent pas la possibilité de la mémorisation d' une information abstraite, qui aura elle-même un rôle dans la catégorisation. Ils sont de deux types : soit ils tentent d' associer les contraintes des modèles de l' exemplaire et des modèles du prototypeKnapp \& Anderson , 1984; Malt, 1989; Medin, Altom, \& Murphy, 1984), soit celles des modèles de l' exemplaire et de la théorie classique (Allen \& Brooks, 1991; Medin, Dewey, \& Murphy, 1983; Smith, Langston, \& Wisbett, 1992; Smith, Patanalo, \& Jonides, 1998).

De façon générale, pour les modèles de l' exemplaire, une question cruciale est bien celle de savoir si un traitement basé sur de simples mécanismes associatifs portant sur des éléments d' information peut être à même de rendre compte des catégories sémantiques quelles qu' elles soient, c' est-à-dire de rendre compte, à un moment ou à un autre, de différences de nature, y compris sur le plan ontologique.

Les prédictions soulignent en effet que des patterns typiques, reflétant des organisations différentes de propriétés, émergent mécaniquement, et qu' il serait possible d' y reconnaître des profils propres à chaque catégorie ontologique. Par exemple, les catégories du vivant correspondraient à des patterns d' activation de nombreuses propriétés fortement corrélées entre elles et de quelques propriétés 
distinctives supports de discrimination. Par contre, les catégories d' objets fabriqués correspondraient à des patterns d' activation de propriétés en petit nombre fortement corrélées entre elles (structure et fonction), qui dessineraient de petits ensembles ayant une forte cohérence interne mais faiblement liés les uns aux autres. Les données de McRae et Cree (2002), Garrard, Lambon-Ralph, Hodges et Patterson (2001) semblent accepter cette interprétation. Rappelons :

- que les modèles théoriques sous-jacents ne sont pas tributaires d' hypothèses sur un fractionnement de la mémoire sémantique (Tyler, Moss, Durrant-Peatfield \& Levy, 2000).

- qu' ils ne sont pas davantage tributaires d' hypothèses quant à la nature des propriétés en jeu.

Or il est important de préciser que les organisations différentes qui émergent "mécaniquement" ne peuvent être caractérisées que statistiquement, et, par là, les inférences quant à la nature ontologique de la catégorie correspondante émergeante ne peuvent être formulées qu' en probabilité. Dans les données obtenues paMcRae et Cree, par exemple, si l' on se focalise sur l' organisation et le recouvrement des propriétés (p.222 et 236), on ne trouve pas de frontières franches entre des distributions de propriétés renvoyant à des catégories d' appartenance ontologique distincte, mais bien une présence plus ou moins probable de catégories dans tel ou tel type d' organisation. A ce stade, il semble donc difficile de faire l' économie de facteurs tels que la nature des catégories ou celle de leurs propriétés.

Ces modèles, nonobstant toutes les qualités qu' ils présentent, nous semblent donc malhabiles à approcher précisément les questions liées aux différences sémantiques ou ontologiques entre catégories, s' il s' avère que celles-ci ne peuvent pas être négligées dans l' étude des processus de catégorisation. 


\section{Théorie des concepts fondés sur des théories}

Les modèles de l' exemplaire restent fondamentalement basés sur des calculs de similitude, et ceci constitue le cœur de la critique qui sera formulée à leur encontre. Au cours des années 80, une nouvelle conception a vu le jour, qui se propose de caractériser la structure conceptuelle en spécifiant les contraintes qui président à son élaboration. Au cœur de cette conception héritée des idées de la philosophie des sciences sur la signification (Putnam, 1975), le questionnement de fond porte sur les liens profonds qui unissent les exemplaires d'une même catégorie, c'est-à-dire la cohérence conceptuelle. Puisque nous ne formons pas des concepts sur la base de tous les regroupements d'objets possibles, l'étude de ces principes est cruciale pour mettre en évidence comment ceux que nous formons sont mis en relief par l'esprit. Dans le cadre des conceptions antérieures, même si le problème de la cohérence n'est pas abordé per se, celle-ci est rendue par la similitude entre les exemplaires ici fortement remise en cause. La véritable contrainte serait ce qui expliquerait le choix des propriétés, leurs relations, le pourquoi des liens entre exemplaires (Ahn, Gelman, Amsterlaw, Hohenstein, \& Kalish, 2000; Keil, Smith, Simons, \& Levin, 1998; Murphy \& Medin, 1985;

Rehder \& Hastie, 2001). C'est pourquoi cette conception est dite aussi "fondée sur l'explication causale". Ahn (1998) s' attache ainsi à démontrer que la relation qui est généralement trouvée entre le relief des propriétés et la nature ontologique des catégories (propriétés structurales pour les objets naturels, propriétés fonctionnelles pour les objets fabriqués) recouvre de fait le rôle éminemment causal de ces propriétés dans leur catégorie respective; elles y jouent le rôle de cause pour d' autres propriétés. Dans cette expérienceAhn propose à des adultes 
de juger des phrases exprimant un lien causal entre deux propriétés : " un oiseau a des ailes parce qu' ikole "versus " un oiseau vole parce qu' id des ailes ". Les sujets jugent plus probable que des propriétés fonctionnelles soient des causes d' autres propriétés pour les objets fabriqués, et que les propriétés structurales soient des causes d' autres propriétés pour les objets naturels.

Trois ensembles d'éléments sont pris en compte par les tenants de cette conception pour rendre compte de la nécessité d'une nouvelle approche des concepts et catégories d'objets : (a) l' appartenance ontologique, (b) la pondération et le relief des traits, (c) l' explication.

a) Négliger de considérer des types de propriétés donne l'illusion que les concepts ont le même type de structure représentationnelle. Or, pour n'évoquer que les concepts sous-jacents aux objets, il semble nécessaire de prendre en compte les distinctions ontologiques entre concepts (Medin, Lynch, \& Solomon, 2000). Certaines de ces distinctions s'appuient d'ailleurs sur la nature des traits partagés (Barton \& Komatsu, 1989; Cordier \& Tijus, 2001; Keil, 1979; Malt \& Johnson, 1992; McRae, de Sa, \& Seidenberg, 1997; Sloman \& Malt, 2003). Les concepts sont élaborés par différents moyens (découvertes, expériences versus convention) et une liste de propriétés ne peut seule porter cette information. L'information sur l'ontologie des objets entre en jeu pour donner un statut différent aux propriétés représentées (exhaustive et complète, ou non exhaustive et révisable), et sans doute pour les sélectionner.

b) On découvre l'expression cohérence conceptuelle pour la première fois à travers le travail de Murphy et Medin (1985) où ils dressent les prémisses de cette conception, selon laquelle la structure conceptuelle résulte de cette contrainte de 
cohérence. Au contraire, les autres conceptions fondent implicitement la cohérence sur le calcul de similitude. Il est supposé en effet que la similitude va mettre en relief certains regroupements par rapport à d'autres et que nos concepts rendent compte de ces regroupements. Cependant, juger que des objets sont semblables n'explique en rien pourquoi ils le sont et un contre-argument valide consisterait à dire que les objets apparaissent semblables justement parce quils sont connus comme appartenant à une même catégorie. Les expériences de Sloustky et Lo (1999), Sloutsky et al. (2001) sont particulièrement intéressantes quant au rôle que peut prendre à cet égard une dénomination commune. En d'autres termes, la similitude permet d'évoquer la cohérence et non de l'expliquer. D'autant que des regroupements d'objets dissemblables peuvent être tout à fait cohérents, et même stockés sous certaines conditions en mémoire. C'est le cas des objets composant les catégories ad hoc (Barsalou, 1983, 1985). Ainsi, le regroupement \{réveil, carnet d'adresse, chemise $\}$ apparaît tout à fait cohérent dans le contexte de la préparation d'une valise.

Ce dernier exemple permet de mettre en évidence l'insuffisance d'une théorie conceptuelle fondée sur le partage de traits. En effet, dans ces catégories ad hoc, ce qui permet de réunir les objets peut ne pas apparaître dans la description même des objets. Si l'on demande à des sujets de lister l'ensemble des propriétés d' un réveil, il est fort peu probable que la propriété " à emporter en voyage " soit mentionnée. Plus généralement, c'est le problème de la sélection et la pondération des traits qui est posé ici. La pondération des traits est fonction du nombre d'exemplaires d'une même représentation conceptuelle qui possède ce trait. Les traits fréquents contribuent à augmenter l'air de famille des souscatégories et donc leur typicalité. Or, même si le trait " forme en demi-lune " 
caractérise la plupart des exemplaires des concepts banane et boomerang, les sujets de Medin et Shoben (1988) ont jugé les boomerangs droits moins typiques de la catégorie des boomerangs que les bananes droites dans la catégorie des bananes. Ceci s'explique par le fait que les traits d'une représentation conceptuelle n'ont pas le même relief ou saillance. La pondération va prendre en considération ce relief, de concert avec la fréquence. Ce relief est susceptible de varier selon les contextes (Denis \& Le Ny, 1986; Murphy \& Medin, 1985). Ainsi, pour les boomerangs, si le but est d'acheter un étui pour le ranger, ce trait sera plus important que si l'on recherche un boomerang pour faire un cadeau. Si la caractéristique " inflammable " des billets de banque n'est pas prépondérante au supermarché, elle le devient dès lors que le problème à résoudre est de faire un feu par tous les moyens. Pour terminer, il faut ajouter qu’à la sélection et la pondération des traits s'ajoute le problème des relations entre les traits. Une perspective qui conçoit les traits d'une représentation conceptuelle comme indépendants serait caduque dans la plupart des cas (Komatsu, 1992; Medin \& Wattenmacker, 1987). La plupart des concepts ne sont en effet pas réductibles à une liste de traits indépendants, ni même à des traits corrélés.

c) Plus radicalement, l' hypothèse est ici que les concepts sont plus que la somme des traits qui les composent. Il ne suffirait pas de prendre toutes les propriétés d'un oiseau, comme pond des aufs, a des ailes, a un bec, vole... pour avoir un oiseau car dans le cas où l'on rencontrerait une entité qui possède toutes ces propriétés mais qui pondrait parce qu’il vole, on serait peu enclin à juger quỉl s'agit d'un oiseau réel, mais plutôt d'un oiseau de Science Fiction. Ainsi, un concept est caractérisé surtout par des relations causales spécifiées entre les traits. C' est ce que souligne clairement une expérience de Murphy eAllopenna (1994), 
qui présentent aux participants de leur étude deux types de catégories : intégrées (avec les propriétés suivantes: construit en Afrique, habitacle ouvert, vert, utilisé dans la savane, a des roues) et neutres (vert, transmission manuelle, pneus radiaux, sièges en vinyle, airbags). Les essais nécessaires pour classer les items correctement sont beaucoup plus faibles pour les catégories intégrées. Le nom de la catégorie n' est jamais prononcé. C' est la présence ou pas d' un "ciment" entre les propriétés qui provoque une différence de traitement. L' expérience dRips (1989) évoquant des pizzas et des pièces de monnaie, en témoigne également.

Les dissociations entre catégorisation et similarité d' une part et l' impact de connaissances préalables d' autre part ont été mis en évidence également par Barrett, Abdi, Murphy et Gallagher (1993), Hayes, Taplin et Longstaff (2002), Heit (2001), Heit et Bott (2000), Murphy et Allopenna (1994), Pazzani (1991), Thibaut, Dupont et Anselme (2002). Ainsi, nos représentations conceptuelles pourraient souligner, outre la nature des traits importants, des informations explicatives sur les transformations possibles de ces traits, transformations probablement liées à nos connaissances dans des domaines autres que celui sur lequel porte la catégorisation.

\section{Les différentes fonctions des concepts}

Nous avons déjà évoqué la perspective de Barsalou (1991) qui distingue, parmi les fonctions des concepts, la catégorisation et la conceptualisation. La catégorisation, qui nous permet d’identifier les objets (leur apparence et leur type) et de les distinguer des membres des autres catégories rend compte d'une organisation de la mémoire. Cependant, catégoriser n'est pas une fin en soi et la connaissance des propriétés importantes d'un objet et de sa catégorie 
d'appartenance donne accès à des informations indispensables si l'on veut interagir avec cette entité, l'intégrer dans nos actions et nos planifications. Aussi la structure des concepts et catégories ne peut-elle être restreinte à une simple abstraction définitive et fixée de propriétés jugées importantes.

On peut dresser une liste non exhaustive des fonctions remplies par les concepts (Medin \& Coley, 1998; Medin \& Heit, 1998; Solomon, Medin, \& Lynch, 1999; Tijus \& Cordier, 2003). Outre la catégorisation, qui est une procédure qui permet d’identifier le nouveau en le connectant à l'ancien, le concept ou la catégorie identifiés apportent au système cognitif des connaissances stockées relatives au type, à la fonction, à la structure, au comportement... de l'objet en question, connaissances qui vont être le support des autres fonctions. La représentation conceptuelle doit permettre donc de faire des prédictions sur les caractéristiques pertinentes de l'objet, comme son comportement dans une situation donnée (fonction d'inférence). Elle contient également des informations cruciales pour l'aboutissement d'un raisonnement, la compréhension et l'explication. Catégoriser un objet singulier comme étant une motocyclette $125 \mathrm{CC}$ permet de repérer les éléments importants et de fournir une raison appropriée de ce qu'un mineur la conduise. Les fonctions d'explication et de compréhension sont cruciales également pour comprendre et expliquer (et non plus seulement savoir) pourquoi les membres d'une même catégorie vont ensemble. La représentation conceptuelle doit pouvoir être révisée et mise à jour (fonction d'apprentissage) au fur et à mesure que de nouveaux éléments y sont placés (Heit \& Bott, 2000). Dans cette recherche, les expérimentateurs font apprendre à leurs sujets des catégories nouvelles, des bâtiments (architecture " Jugenstil ") et des véhicules. Dans la mesure où les connaissances préalables sont importantes pour 
ces catégories, ils analysent l' apprentissage dans son déroulement, au fur et à mesure que les exemples présentés augmentent en nombre, afin de voir si les stratégies du sujet sont affectées et de quelle manière par la succession des stimuli. Dans les étapes ultimes de l' apprentissage, le rôle des traits critiques des nouveaux exemplaires devient de plus en plus net, par opposition aux traits de remplissage, témoignant de la discriminabilité acquise de la nouvelle catégorie sur l' arrière-plan des connaissances anciennes. Les concepts sont également une grande partie des contenus des messages que l'on émet à l'attention d'un interlocuteur (fonction de communication). La prise en compte d'éléments extraconceptuels comme le but de la communication et le niveau de l'interlocuteur permet de faire référence à la même entité de diverses manières (une motocyclette 125CC est également un véhicule). Enfin, toujours dans le domaine du langage, les concepts permettent de comprendre les expressions, les combinaisons de mots et de résoudre les ambiguités (fonction de combinaison conceptuelle). C'est ainsi que nous pouvons comprendre dans la plupart des contextes " la réparation du garagiste " comme la réparation effectuée par le garagiste, non pas comme la réparation d'un garagiste défaillant.

On pourrait penser que ces différentes fonctions s'exercent sur des structures conceptuelles différentes. Si l'on demande par exemple à des sujets adultes de sélectionner parmi un ensemble d'arbres les meilleurs exemples, on peut penser qu'ils vont sélectionner les arbres qui représentent le mieux la catégorie, les arbres (proto)typiques. Il s'agit pour eux d'une épreuve de catégorisation banale et ils n'ont qu'à classer. Or, la même tâche administrée à des experts donne des résultats différents. Ceux-ci font leurs choix en fonction de critères idéaux (Barsalou 1985, 1987) comme la hauteur maximale et l'absence de 
mauvaises herbes environnantes, et non les valeurs moyennes des critères. Tout se passe comme si les experts planifiaient la construction d'une forêt saine, les critères pris en compte relevant de la santé des arbres. Ainsi, la structure interne des catégories, ou des ensembles d'objets, est largement contrainte par d'autres fonctions que la catégorisation (Johnson \& Mervis, 1998; Medin et al., 2000).

Il ressort de tout ce qui précède qu'une nouvelle théorie des concepts est nécessaire, qui prendrait en compte les propositions validées des théories précédentes, mais qui dépassent certaines de leurs limites. Cette conception se doit de montrer que la cohérence de structure conceptuelle n'est pas toujours fondée sur la similitude, ni sur le partage de propriétés indépendantes ou corrélées et pondérées, mais également sur l'explication des relations entre les propriétés, sur leurs transformations et leurs opérations possibles. Elle doit montrer encore que la catégorisation ne consiste pas toujours dans un appariement d'attributs. Elle doit mettre en évidence que les propositions des conceptions anciennes restent valables, mais seulement pour certains types de concepts, de contextes, de fonctions conceptuelles. Les concepts seraient plus riches et plus flexibles que les anciennes conceptions ne permettaient de l'envisager. Cette flexibilité est gérée par les connaissances d'arrière-plan et des croyances que les sujets se construisent sur le monde. Les concepts ne sont pas des entités isolées mais bien intégrées dans des systèmes de croyances, les théories naïves, qui les contraignent, les enrichissent et les rendent cohérents.

Les concepts sont intégrés dans des systèmes d'explication causale, des théories

Ces conceptions partent de la nécessité, pour spécifier la structure conceptuelle, d'inclure les connaissances et croyances d'arrière-plan sur le monde 
(Ahn, 1998; Ahn, Kim, Lassaline, \& Dennis, 2000; Keil, 1989; Komatsu, 1992;

Murphy \& Medin, 1985; Rehder \& Hastie, 2001). C'est pourquoi cette conception est également dite fondée sur l'explication. Les théories apportent une information cruciale dans trois domaines :

- une information relative aux traits qui composent les concepts, leur identité, leur statut (nécessaires et suffisants, ou seulement critiques). Les théories permettent également de sélectionner les bonnes corrélations entre les traits et d'expliquer leurs relations fonctionnelles et causales. Ainsi, pour le concept oiseau, les théories précisent que le trait " voler " est corrélé au trait " a des ailes ", et qu' ils sont liés de manière causale. Elles expliquent que les traits qui caractérisent l'aspect et le comportement des oiseaux, sont les manifestations d'une même cause, comme la structure génétique.

- une information relative à la cohérence, aux relations qui lient les exemplaires d'un même concept. Les théories permettent de comprendre dans quelles situations la similarité peut suffire et dans quelles situations il est nécessaire d' invoquer d'autres principes.

- une information relative aux relations entre un concept donné et tous ceux auxquels il peut être lié, en précisant son rôle dans certains événements. Ainsi, le concept oiseau peut inclure le fait que les oiseaux mangent des graines, vivent parfois dans des cages...

Toutes ces connaissances relationnelles sont liées et s’influencent mutuellement. Elles forment un réseau de relations complexes qui situe le concept donné dans la base de connaissances de l’individu, comme l'explique clairement Keil (1989) dans cette citation. 
«La plupart des concepts sont eux-mêmes des théories partielles dans ce qu'elles contiennent des explications des relations de leurs constituants, de leurs origines, et de leurs relations avec d'autres clusters de traits. Ceci est (...) plus important encore pour les concepts d'objets puisqu'un concept d'objet bien spécifié dépend non seulement de la compréhension des relations causales entre ses propriétés et des raisons de l'existence de corrélations particulières, mais également des rôles causaux potentiels que joue cet objet de manière régulière et de manière stable lorsqu'il interagit avec d'autres objets » (p. 281).

Pour mettre en évidence la validité de cette conception par rapport aux autres, les auteurs se sont attachés à étudier le rôle crucial des connaissances d'arrière-plan dans les activités qui engagent les représentations conceptuelles, notamment l'apprentissage de concepts (Hayes, Taplin, \& Longstaff, 2002; Heit, 1997; Heit \& Bott, 2000; Medin \& Wattenmaker, 1987; Medin, Wattenmaker, \& Hampton, 1987; Murphy \& Allopenna, 1994). Ces auteurs ont montré que les nouveaux concepts sont appris en référence à un ensemble de connaissances que les individus possèdent dans le même domaine. Au cours d' épreuves de catégorisation de nouveaux objets, les performances sont améliorées lorsque les liens entre les concepts à acquérir et une partie pertinente des connaissances d'arrière-plan, ou lorsque les principes sous-jacents sont clairement explicités. La supériorité de ce type de conceptions sur les conceptions antérieures a été mise en évidence notamment par Medin, Wattenmaker et Hampton (1987) qui ont donné l'occasion à leur sujet de classer des dessins dans différentes catégories, structurées selon les critères de la conception de l' air de famille. En utilisant un matériel et des consignes variés, ils soulignent que si les propriétés sont présentées comme indépendantes, le classement des participants est 
unidimensionnel. Lorsque les propriétés sont présentées en lien, le traitement unidimensionnel est abandonné, et ceci avec d' autant plus de force que les propriétés présentent des liens de causalité. Globalement, (1) les performances sont meilleures lorsque les relations entre les propriétés sont apparentes, (2) l' explication causale a de meilleurs effets que læovariation de propriétés (Ahn, Kalish, Medin, \& Gelman, 1995). Il est symptomatique de cet état de chose que le matériel construit pour mettre en évidence le rôle des cadres explicatifs est riche de relations causales explicites (parce que, donc, en conséquence : Rehder \& Hastie, 2001). Des analyses expérimentales plus fines portent sur l' efficacité des relations de causalité entre propriétés. Par exemple, les conceptions de Ahn (1998) s' opposent à celles dRehder (2003), Rehder \& Hastie (2001, 2004) sur un point : alors que les seconds privilégient la " densité causale " (le fait qu' une propriété soit indifféremment l' effet ou la source de relations causales)Ahn considère que c' est le statut de cause joué par une propriété qui lui conférera un rôle premier, et aura des incidences sur la catégorisation (Ahn, Gelman, Amsterlaw, Hohenstein, \& Kalish, 2000; Ahn, Kim, Lassaline, \& Dennis, 2000).

Il existe plusieurs variantes de cette conception dans des domaines divers de la cognition. Pour ce qui est de la représentation conceptuelle chez l'adulte (Ahn, Kalish, Gelman, Medin, Luhmann, Atran, Coley, \& Shafto, 2001; Barton \& Komatsu, 1989; Lakoff, 1987; Medin \& Ortony, 1989; Medin \& Wattenmaker, 1987; Murphy \& Medin, 1985; Rips, 1989), nous l’illustrerons brièvement par l'approche qualifiée d' essentialisme psychologique (Medin \& Ortony, 1989; mais voir Braisby, Franks, \& Hampton, 1996; Strevens, 2000), représentative de cette communauté d’idées et qui a aussi donné lieu à des hypothèses spécifiques dans le domaine du développement conceptuel (par exemple, Carey, 1985; Keil, 1989). 
Cette approche est par ailleurs la moins "parcimonieuse", au sens où elle est celle qui met en avant les hypothèses les plus fortes dans l' interprétation des processus de catégorisation.

Selon cette conception, les théories (croyances) et connaissances d'arrièreplan conduisent les individus à élaborer leurs représentations conceptuelles comme si elles possédaient une essence qui fait que ces choses sont ce qu'elles sont. Ces connaissances permettent de sélectionner les informations qui vont constituer le lien essentiel entre les exemplaires du concept. Cette essence postulée est une explication cachée, profonde de la cohérence catégorielle. Elle n'est pas facilement accessible et ne peut par conséquent bien souvent être utilisée comme telle dans l'activité de catégorisation. C'est pourquoi on fait l' hypothèse que la catégorisation va se fonder sur des propriétés plus accessibles, souvent de nature perceptive, mais contraintes par l'essence (à la manière dont le phénotype de l'oiseau est contraint par son génome) (Keil, 1994). Lors de l'élaboration des concepts, ces propriétés accessibles sont les premières discriminées et acquises et la compréhension du concept va consister dans l'établissement des liens causaux unilatéraux entre traits accessibles et profonds. Ces liens causaux sont les conditions à respecter pour qu'un nouvel objet entre dans une catégorie donnée.

L' hypothèse $\mathrm{de'}$ essentialisme psychologique a été proposée paMedin \& Ortony (1989) en rupture avec l' hypothèse philosophique du même nom. Cette dernière présente effectivement - notamment pour le chercheur en psychologie des problèmes redoutables en ce qu' elle dissocie le contenu du concept de la détermination de la référence (Braisby et al., 1996; Pothos \& Hahn, 2000). L' essentialisme psychologique ne statue pas sur l' existence d' une essence. Il pose 
simplement que les individus ont une telle croyance, et que cette croyance est critique pour les jugements d' appartenance catégorielle. L' essence se trouve donc approchée par l' essentialisme psychologique d' une manière moins ambitieuse. Cette hypothèse est limitée à la catégorie ontologique des choses de la nature. A notre connaissance, elle a été testée avant tout sur des choses vivantes, animées ou non (animaux et plantes) à l' exclusion des roches et minéraux.

Cette position est-elle un retour déguisé de la conception classique, la croyance essentialiste tenant lieu de propriétés nécessaires et suffisantes? Nous ne sommes pas réellement en présence d' une définition de la catégorie, puisque l' essence n' est pas isolée, voire même complètement inconnue. Elle ne peut jouer le rôle d' une propriété nécessaire ET suffisante. Toutefoişothos et Hahn (2000) examinent son rôle comme propriété nécessaire OU suffisante.

Le statut de propriété nécessaire implique de tester si la croyance en une propriété "profonde" décide de l' appartenance catégorielle, et ceci indépendamment de fluctuations contextuelles éventuelles. Or, Braisby et al. (1996), Pothos et Hahn (2000) ${ }^{5}$ s' accordent pour reconnaître que les individus peuvent mettre en auvre des raisonnements essentialistes, mais qu' ils les réservent à certains contextes : un essentialisme optionnel en quelque sorte, parmi d' autres possibilités. Il est difficile dans ces conditions d' accorder le statut de propriété nécessaire à cette croyance, mais, possiblement, celui d' une propriété suffisante, laissant ouverte la possibilité de décider de l' appartenance catégorielle d' un exemplaire donné qui ne serait pas par ailleurs crédité de cette essence.

\footnotetext{
${ }^{5}$ Dans cette recherche, les auteurs proposent à leurs sujets de petits scénarios sur une créature imaginaire et une créature bien connue. Des variantes contextuelles sont introduites dans le scénario sur les origines de la créature imaginaire. Finalement, les participants doivent juger de la ressemblance entre la créature imaginaire et la créature réelle. On observe que les variantes introduites dans le scénario changent la nature des réponses.
} 
Le raisonnement essentialiste apparaît dès lors comme une expression extrêmement "affaiblie" d' un raisonnement scientifique d' expert. Mais il est bien entendu qu' il n' y a pas lieu d' assimiler une croyance à une connaissance scientifique, et qu' il est impossible de parler réellement d' expertise. Dès lors, nous pouvons nous poser la question de l' extension du raisonnemenessentialiste chez les individus. Pourquoi le lier de façon restrictive au monde du vivant ou au monde de la nature ? N' est-ce pas faire indirectement allégeance à' essentialisme philosophique ? Rotschild et Haslam (2003) donnent des exemples de raisonnements essentialistes hors du domaine ontologique des objets de la nature, par exemple au sein du monde social, ou de la catégorie ontologique des objets fabriqués. Dans certaines situations (objets bénis, fétichisme), des raisonnements qui paraissent de même nature seraient mis en auvre (même si, parallèlement, on souscrit à l' attachement privilégié des raisonnementessentialistes aux objets de la nature). Ceci traduirait alors un fonctionnement psychologique plus général que ce à quoi il était confiné initialement.

De nombreux enjeux restent donc sans réponse claire à l' heure actuelle quant à l' hypothèse dé essentialisme psychologique et à sa portée, et ils dessinent des orientations à prendre dans un avenir proche :

- a minima, il est nécessaire d' expérimenter sur l' ensemble des objets qui relèvent du monde de la nature (y compris sur le "règne minéral") et non pas seulement sur le biologique, pour isoler éventuellement cette croyance en une essence qui serait aux fondements des processus de catégorisation; - existe-t-il une extension d' un raisonnemenessentialiste à d' autres domaines ontologiques, et quelles sont les conditions qui favorisent cette perméabilité entre domaines ontologiques différents? 
- quelle est la "profondeur", la "force" de cette croyance ? A quelles conditions (ou dans quels contextes) peut-elle être mise en défaut?

Ces enjeux nous semblent importants, même si leur étude peut s' avérer délicate. Dans l' état actuel des choses, une hypothèse minimale sur la croyance en l' existence de liens de causalité entre propriétés "cachées" et propriétés de surface telle que celle proposée par Strevens (2000; 2001) nous semble prudente. Elle permet de mettre en valeur ce qui nous semble un des enjeux majeurs de la théorie fondée sur les théories : la recherche de relations causales entre propriétés, selon la nature de celles-ci. Cette hypothèse minimale peut s' inscrire éventuellement dans le cadre d' une hypothèsessentialiste. Elle peut également s' inscrire avec quelques aménagements - nous semble-t-il - dans le cadre d' hypothèses téléologiques. Dans ce cadre, les entités sont considérées comme destinées à un but (Kelemen, 1999). C' est donc un raisonnement d' ordre fonctionnel qui se trouve appliqué au premier chef.

Un débat théorique semble là nécessaire. Un indicateur qui permet - nous semble-t-il - de nuancer une visée hégémonique de poser les problèmes est le lien qui est fait - ou non - entre la profondeur causale et le relief de la propriété (Ahn, Kim, Lassaline, \& Dennis, 2000). Nous faisons l' hypothèse que ce lien n' est pas systématique, mais est fonction de la nature de la tâche proposée aux participants. En effet, lorsqu' on demande à un individu de faire des inférences sur tel ou tel type d' objet, par exemple sur l' ensemble des propriétés qui concourent à la qualité d' un produit, et sur le réseau de causalité qui sous-tend cette propriété érigée en but à atteindre (Dompnier, Cordier, Kirsche, \& Lescop, 2005), alors les propriétés qui prennent le plus de relief sont les plus superficielles, alors que dans le même temps les propriétés causales sont créditées de peu de poids. Autrement dit, les 
propriétés reconnues comme causalement responsables de la qualité ont peu de poids au regard des propriétés qui expriment immédiatement cette qualité. Dans ce cas, il nous semble qu' un raisonnement de nature téléologique se trouve bien exprimé par l' individu dans son comportement. Il est évidemment nécessaire de généraliser ce type d' études à d' autres catégories ontologiques. Mais cela indique qu' il est important de dissocier l' étude du type de raisonnement appliqué aux jugements catégoriels - essentialiste , téléologique - de l' étude du réseau causal sous-jacent, de la densité causale (Rehder \& Hastie, 2001).

En attendant, il nous paraît fondé de croire que le raisonnement "essentialiste" est - à tout le moins - optionnel chez les individus, et limité à des tâches où les distinctions ontologiques pourraient être porteuses d' informations critiques.

En résumé, au sein des théories de la catégorisation fondées sur les théories, la représentation conceptuelle n'est plus isolée, mais située dans un ensemble de connaissances et de croyances plus ou moins liées au domaine ciblé. L'organisation conceptuelle a ici la forme d'un réseau de relations causales (Medin \& Wattenmaker, 1987; Rehder \& Hastie, 2001) dans lequel de nombreux liens sont susceptibles d'être activés en fonction du contexte et de la fonction conceptuelle actualisée. Les concepts sont des mini-théories (Keil, 1989) qui sont elles-mêmes emboîtées dans les théories que les sujets construisent sur le monde. Les concepts sont définis en fonction de la manière dont fonctionne le monde.

Les théories fournissent un cadre explicatif qui souligne les raisons qui permettent de lier des exemplaires d'un même concept ou de concepts différents. Les caractéristiques propres aux objets deviennent bien moins importantes pour la 
catégorisation que ne l'est la manière dont ils peuvent s’intégrer dans ce cadre explicatif.

Il ressort de tout ceci que les concepts englobés dans des théories rendent compte à la fois de l'organisation schématique et en catégories. La différence avec les autres conceptions réside dans le fait que les organisations sont plus riches, plus flexibles, et que selon la tâche, le contexte, les connaissances et les croyances, les fonctions conceptuelles actualisées, les structures et les relations évoquées seront différentes. Pour l'organisation en catégories, la taxonomie est organisée autour des connaissances ontologiques qui ont une influence cruciale sur l'élaboration du concept. Quant à l'organisation schématique, rappelons que selon certains auteurs, les schémas et les théories sont le même type de corps organisé de connaissances (Komatsu, 1992; Lakoff, 1987; McCauley, 1987; Neisser, 1987), les premiers étant contextuellement restreints à un type de situations et n’intégrant pas des explications sur la manière dont les attributs sont reliés. Ces deux types de structures sont certes des modèles cognitifs idéalisés (Lakoff, 1987) parce qu’ils sont définis en rapport à des situations idéalisées, les théories incarnant les modèles les plus complexes et les plus élaborés. On peut alors penser que les schémas forment une partie intégrante des théories.

Une faiblesse de cette approche théorique à l' heure actuelle vient de l' imprécision, du flou, qui entoure les connaissances d' arrière-plan qui sont évoquées de façon récurrente dans cette approche de la catégorisation. Elles se trouvent généralement reliées à un domaine (domaine du psychologique, domaine du biologique, domaine de la physique, pour les trois domaines qui sont couramment évoqués), et qualifiées de naïves pour bien les distinguer des savoirs scientifiques élaborés dans d' autres circonstances, mais sans que leur contenu soit 
précisément cerné : un certain recours globalisant à ces savoirs naïfs peut apparaître de l' ordre de l' incantation. De plus, on connaît encore mal les mécanismes et les conditions d' apprentissage de ces contenus et cela reste un enjeu majeur pour une connaissance fine de la conceptualisation et du concept. Si par contre on se tourne vers les acquis expérimentaux, ils sont nets sur le plan de la connaissance du rôle joué par les liens entre propriétés, et sur celui de l' influence demini-théories sur l' apprentissage. Des ambitions plus restreintes, mais qui prouvent leur heuristique.

\section{Les évolutions paradigmatiques des conceptions du concept au travers du prisme de la neuropsychologie: le cas des déficits catégoriels spécifiques}

Notre examen du champ théorique des concepts souligne les interrelations entre les différentes approches et leur complexité: la présence de modèles mixtes de la catégorisation est un premier indicateur qu' il n' existe pas UNE théorie de la catégorisation, consensuelle à ce jour, mais que selon les consignes, le matériel, la nature de l' apprentissage (intentionnel ou incident), l' un ou l' autre modèle sera plus à même de rendre compte de manière satisfaisante des faits enregistrés. Cet éclectisme n' est pas exceptionnel dans le champ de la psychologie et existe pour d' autres secteurs. Notre intention est maintenant d' examiner et de nous interroger sur les lignes de force dessinées par ces modèles théoriques, appliquées au champ de la neuropsychologie, et en particulier au sein des recherches qui se focalisent sur les déficits catégoriels spécifiques. La contribution de la psychologie cognitive dans le développement de ce champ de recherche est majeure. Si les 
progrès de la neuropsychologie permettent de poser en des termes nouveaux la question des rapports entre pensée et cerveau, ils nous paraissent confirmer également les besoins de la recherche neuropsychologique en des constructions théoriques validées par des programmes expérimentaux conséquents (Tiberghien, 1999).

Le profond intérêt pour les déficits catégoriels spécifiques qui touchent certains patients cérébro-lésés vient de ce que l' on pense pouvoir y trouver, d' une manière relativement transparente, un paysage - en négatif - de l' organisation des informations catégorielles en mémoire. Il est en effet troublant de voir des patients qui présentent des détériorations spécifiques pour la dénomination ou la description de choses vivantes, ou de choses inanimées (voir Marques, 2002, pour une recension récente). Cependant, les déficits enregistrés n' ont pas de frontières nettes et varient de façon importante selon les patients. Par exemple, certains montrent des déficits pour les fruits, les végétaux, mais pas les animaux (Hillis \& Caramazza, 1991); d' autres pour la nourriture, les pierres précieuses et les instruments de musique, alors que les choses vivantes sont préservées (Silveri \& Gainotti, 1988). La complexité des faits laisse en compétition au moins trois grandes hypothèses ${ }^{6}$, qui trouvent leurs sources dans différentes conceptions de la structure catégorielle.

La première hypothèse qu' il convient d' examiner est celle d' un stockage par catégorie, celle-ci étant considérée dans son extension. Cette hypothèse a été rapidement écartée sous cette forme pour deux raisons (McRae \& Cree, 2002) : la première raison est la difficulté d' envisager les choses sous l' angle d' une localisation cérébrale - l' approchøeuronale privilégiant une organisation basée

\footnotetext{
${ }^{6}$ Nous n' évoquerons pas leurs variantes.
} 
sur la fonction. La deuxième raison concerne l' allure des données, et en particulier leur grande hétérogénéité. Il est donc plus intéressant d' adopter d' emblée une vision des catégories en intension, et de parler en terme de traits, de leur nature, de leur relief, de leur recouvrement, ce qui peut conduire à privilégier un modèle distribué de l' information catégorielle.

Warrington et Shallice (1984) proposent de considérer la dissociation vivant / non vivant observée de façon assez fréquente chez les patients comme un sousproduit de la différence de relief dans le traitement des traits sensoriels versus fonctionnels dans l' identification de la catégorie. Une hypothèse plus fine a été avancée plus récemment par Gonnerman, Andersen, Devlin et Seidenberg (1997) mettant l' accent sur les corrélations possibles entre propriétés, et en particulier les liens forme et fonction. Les relations forme - fonction permettraient de distinguer les entités vivantes des objets fabriqués. Ces derniers se ressembleraient peu entre eux, leurs formes distinctes étant associées aux fonctions différentes qu' ils doivent réaliser. Au contraire, les entités vivantes seraient plus semblables entre elles, et les variations de forme (par exemple la grande variété des chiens) seraient sans signification fonctionnelle évidente, sauf si des fonctions biologiques sont en jeu (pattes ou ailes pour la locomotion). La plus grande similitude des entités vivantes par rapport aux objets fabriqués, et le taux notable de propriétés corrélées qui y sont présentes, ont été confirmés par le recueil des normes mené par McRae \& Cree (2002), même si des catégories dérogent à l' allure générale (bijoux, instruments de musique).

Ces hypothèses écartent la théorie classique - pour qui toutes les propriétés sont homogènes dans leur rôle et leur impact. Par contre, les théories de l' air de famille peuvent être sollicitées : les preuves expérimentales qu' elles ont apportées 
du rôle du relief des propriétés sont nombreuses. Les théories sur l' explication doivent également être invoquées, dans le rôle différent qu' elles accordent aux propriétés selon 1' origine ontologique de la catégorie.

Plus précisément, si un modèle distribué de l' information semble particulièrement heuristique pour rendre compte des hypothèses de Warrington et Shallice (1994) et de celles de Gonnerman et al. (1997), rien dans les théories de l' exemplaire ne permet de supposer un rôle différent des propriétés selon leur nature et selon les catégories. Il paraît indispensable, au sein de processus de type "classification de patterns" d' introduire des processus symboliques de plus haut niveau, qui rendent compte de cette contrainte supplémentaire. Celle-ci permettrait de modéliser de quelle manière une accumulation d' expériences spécifiques - d' exemplaire contextualisés - conduit à une activation préférentielle de traits sensoriels pour les choses vivantes, de traits fonctionnels pour les objets fabriqués. Il semble théoriquement difficile d' introduire l' idée que la perception agit comme un filtre pour sélectionner les propriétés pertinentes : beaucoup de propriétés envisagées dans les déficits catégoriels spécifiques ne dépendent pas d' une perception élémentaire. Nous voyons encore cette fois l' intérêt de développer des modèles hybrides, connexionnistes et symboliques, comme l' est un modèle mixte " air de famille / exemplaire ".

L' hypothèse dœaramazza et Shelton (1998) développe l' idée d' une organisation conceptuelle reposant sur de vastes domaines ontologiques, ceux-ci reflétant eux-mêmes des déterminismes pertinents en fonction de l' évolution, liés à la survie de l' individu et de l' espèce. Cette proposition, en invoquant la part des distinctions ontologiques dans la catégorisation, paraît a priori solliciter davantage les théories fondées sur les théories. Mais de fait, cette hypothèse fait 
intervenir un facteur phylogénétique qui est absent des attendus des théories de la catégorisation, quelles qu' elles soient. Comme le facteur phylogénétique semble un élément central de la proposition de Caramazza et Shelton (1998), un examen plus poussé des apports des théories de la catégorisation nous semble ici tourner court.

\section{Conclusion}

L'organisation conceptuelle s'est trouvée considérablement enrichie et les relations entre les objets diversifiées. Le concept, d'abord conçu comme isolé dans une organisation hiérarchique et défini par des caractéristiques propres, est inséré dans des structures contextualisées, puis dans l'ensemble du domaine des connaissances et croyances. Il est vu aujourd' hui comme une structure très flexible, adaptée à la diversité des compétences, et dont le noyau de signification n'est plus seulement fixé par l'analyse des objets eux-mêmes ou de leur rôle dans un nombre limité d'événements et de situations, mais bien par l'ensemble des connaissances et croyances dans un domaine large.

De fait, plusieurs théories de la catégorisation cohabitent à l' heure actuelle. Basées sur l' étude des représentations mentales pour les premières, elles ont évolué ensuite par le jeu des apports de modèles sub-symboliques, à la fois très puissants - par la généralisation de processus se déployant en parallèle - mais cognitivement réducteurs - par le recours à des processus de bas niveaux, de type " classification de patterns ". Ces modèles, par ailleurs, ne semblent pas capables de rendre compte pleinement des spécificités de traitement induites par la nature des catégories ou des propriétés. On comprend alors l' intérêt des systèmes 
hybrides où les informations symboliques - sémantiques - sont réintroduites, et ceci malgré les difficultés théoriques et méthodologiques que cela pose. 
Bibliographie.

Ahn, W.K. (1998). Why are different features central for natural kinds and artifacts? The role of causal status in determining feature centrality. Cognition, 69, 135-178.

Ahn, W.K., Gelman, S.A., Amsterlaw, J.A., Hohenstein, J., \& Kalish, C.W. (2000). Causal status effect in children' sategorization. Cognition, 76, B35-B43.

Ahn, W.K., Kalish, C., Gelman, S.A., Medin, D.L., Luhmann, C., Atran, S., Coley, J.D., \& Shafto, P. (2001). Why essences are essential in the psychology of concepts. Cognition, 82, 59-69.

Ahn, W.K., Kalish, C.W., Medin, D.L., \& Gelman, S.A. (1995). The role of covariation versus mecanism information in causal attribution. Cognition, 54, 299 - 352.

Ahn, W.K., Kim, N.S., Lassaline, M.E., \& Dennis, M.J. (2000). Causal status as a determinant of feature centrality. Cognitive Psychology, 41, 361-416.

Allen, S.W., \& Brooks, L.R. (1991). Specializing the operation of an explicit rule. Journal of Experimental Psychology : General, 120(1), 3-19.

Baddeley, A. (1992). La Mémoire Humaine : Théorie et Pratique. Grenoble : Presses Universitaires de Grenoble.

Barrett, S.E., Abdi, H., Murphy, G.L., \& McCarthy Gallagher, J. (1993). Theory-based correlations and their role in children' s conceptsChild Development, 64, 15951616.

Barsalou, L.W. (1983). Ad hoc categories. Memory and Cognition, 11, 211-227.

Barsalou, L.W. (1985). Ideals, central tendency, and frequency of instantiation as determinants of graded structure in categories. Journal of Experimental Psychology: Learning, Memory and Cognition, 11, 629-653. 
Barsalou, L.W. (1987). The instability of graded structure: Implications for the nature of concepts. In U. Neisser (Ed.), Concepts and conceptual development. Ecological and intellectual factors in categorization (pp. 101-140). Cambridge, Cambridge University Press.

Barsalou, L.W. (1990). On the indistinguishability of exemplar memory and abstraction in category representation. In T. K. Srull \& S. Wyer (Eds), Advances in social cognition: Volume III. Content and process specificity in the effects of prior experiences (pp. 61-88). Hillsdale, NJ: Erlbaum.

Barsalou, L.W. (1991). Cognitive Psychology: An Overview for Cognitive Scientists. Hillsdale, NJ: Erlbaum.

Bartlett, F.C. (1932). Remembering: A study in experimental and social psychology. Cambridge: Cambridge University Press.

Barton, M.E., \& Komatsu, L.K. (1989). Defining features of natural kinds and artifacts. Journal of Psycholinguistic Research, 18, 433-446.

Braisby, N., Franks, B., \& Hampton, J. (1996). Essentialism, word use and concepts. Cognition, 59, 247-274.

Brooks, L. (1978). Nonanalytic concept formation and memory for instances. In E. Rosch \& B.B. Lloyd (Eds), Cognition and categorization (pp. 169-211). Hillsdale, NJ : Erlbaum.

Brooks, R.A. (1991). Intelligence without representation. Artificial Intelligence, 47, 139-159. 
Bryant, D.J., Tversky, B., \& Franklin, N. (1992). Internal and external spatial frameworks for representing described scenes. Journal of Memory and Language, 31, 74-98.

Caramazza, A., \& Shelton, J.R. (1998). Domain-specific knowledge systems in the brain: The animate-inanimate distinction. Journal of Cognitive Neuroscience, 10, 1-34.

Carey, S. (1985). Conceptual Change in Childhood. Cambridge, MA: MIT Press.

Chi, M., \& Slotta, J.D. (1993). The ontological coherence of intuitive physics. Cognition and Instruction, 10(2,3), 249-260.

Cordier, F. (2002). Les théories conceptuelles. In F. Cordier \& J. François, Langage et catégorisation (pp.125-144). Paris, Hermès.

Cordier, F., \& Cordier, J. (1991). L' application du théorème de Thalès. Un exemple du rôle des représentations typiques comme biais cognitifs. Recherche en didactiques des mathématiques, 11/1, 45-64.

Cordier, F., Cordier, J., \& Es Saïdi, M. (1997). Problem solving tasks concerning solid geometrical figures. The effetcs of typical representations. European Journal of Psychology of Education, 3, 305-314.

Cordier, F., \& Tijus, C. (2001). Object properties: A typology. Current Psychology of Cognition, 20, 445-472.

Denis, M., \& Le Ny, J.F. (1986). Centering on figurative features during the comprehension of sentences describing scenes. Psychological Research, 48, 145152. 
Dompnier, L., Cordier, F., Kirsche, L. \& Lescop, O. (2005). La catégorisation des objets fabriqués. Relief et relations causales entre propriétés. Conférence au colloque "Construction du sens", Montpellier, France.

Estes, W.K. (1986). Array models for category learning. Cognitive Psychology, 18, $500-549$.

Garrard, P., Lambon-Ralph, M.A., Hodges, J.R., \& Patterson, K. (2001). Prototypicality, distinctiveness, and intercorrelation: Analyses of the semantic attributes of living and nonliving concepts. Cognitive Neuropsychology, 18, 125174.

Gluck, M.A., \& Bower, G.H. (1988a). From conditioning to category learning: An adaptive network model. Journal of Experimental Psychology: General, 117, 227247.

Gluck, M.A., \& Bower, G.H. (1988b). Evaluating an adaptive network model of human learning. Journal of Memory and Language, 27, 166-195.

Goldstone, R.L. (1995). Mainstream and avant-garde similarity. Psychologica Belgica, $35,145-165$.

Gonnerman, L., Andersen, E., Devlin, J., \& Seidenberg, M. (1997). Double dissociation of semantic categories in Alzheimer' s diseaseBrain \& Language, 60, 58-60.

Hahn, U., \& Chater, N. (1997). Concepts and similarity. In K. Lamberts \& D. Shanks (Eds.), Knowledge, concepts, and categories (pp. 43-92). Cambridge, MA: MIT Press. 
Hayes, B.K., Taplin, J.E., \& Longstaff, M. (2002). Prior knowledge and exemplar similarity in category learning: Further evidence for their integration. European Journal of Cognitive Psychology, 14(4), 549-571.

Heit, E. (1997). Knowledge and conceptual learning. In K. Lamberts \& D. Shanks (Eds.), Knowledge, concepts, and categories (pp. 7-41). Cambridge, MA: MIT Press.

Heit, E. (2001). Background knowledge and models of categorization. In U. Hahn \& M. Ramscar (Eds), Similarity and Categorization (pp.155-178). Oxford University Press.

Heit, E., \& Bott, L. (2000). Knowledge selection in category learning. In D.L. Murphy, Psychology of Learning and Motivation, 39, (pp.163-199). San Diego, Academic Press.

Hillis, A.E., \& Caramazza, A. (1991). Category-specific naming and comprehension impairment: A double dissociation. Brain and Language, 114, 2081-2094.

Hintzman, D.L. (1986). Schema abstraction in a multiple-trace memory model. Psychological Review, 95, 528-551.

Hintzman, D.L., \& Ludlam, G. (1980). Differential forgetting of prototypes and old instances: Simulation by an exemplar-based classification model. Memory and Cognition, 8, 378-382.

Jagot, L. (2002). Catégories et scripts. In F. Cordier \& J. François (Eds), Catégorisation et langage (pp. 145-163). Paris, Hermès. 
Johnson, K.E., \& Mervis, C.B. (1998). Impact of intuitive theories on feature recruitment throughout the continuum of expertise. Memory and Cognition, 26(2), $382-401$.

Joordens, S., \& Becker, S. (1997). The long and short of semantic priming effects in lexical decision. Journal of Experimental Psychology: Learning, Memory and Cognition, 23, 1083-1105.

Keil, F.C. (1979). Semantic and Conceptual Development: An Ontological Perspective. Cambridge, MA: Harvard University Press.

Keil, F.C. (1989). Concepts, Kinds, and Cognitive Development. Cambridge, MA: Harvard University Press.

Keil, F.C. (1994). The birth and nurturance of concepts by domains: the origins of concepts of living things. In L.A. Hirschfeld \& S.A. Gelman (Eds.), Mapping the mind: Domain specificity in cognition and culture (pp. 234-254). New York: Cambridge University Press.

Keil, F. C., Smith, C., Simons, D., \& Levin, D. (1998). Two dogmas of conceptual empiricism. Cognition, 65, 103-135.

Kelemen, D. (1999). The scope of teleogical thinking in preschool children. Cognition, $70,241-272$

Kirasic, K. C., Siegel, A. W., \& Allan, G. L. (1980). Developmental changes in recognition-in-context memory. Child Development, 51, 302-305.

Knapp, A.G., \& Anderson, J.A. (1984). Theory of categorization based on distributed memory storage. Journal of Experimental Psychology: Learning, Memory and Cognition, 10(4), 616-637. 
Komatsu, L. K. (1992). Recent views of conceptual structure. Psychological Bulletin, $112,500-526$.

Lakoff, G. (1987). Cognitive models and prototype theory. In U. Neisser (Ed.), Concepts and conceptual development: Ecological and intellectual factors in categorization (pp. 63-100). Cambridge, MA: Cambridge University Press.

Lamberts, K. (1994). Flexible tuning of similarity in exemplar-based categorization. Journal of Experimental Psychology: Learning, Memory and Cognition, 20(5), 1003-1021.

Le Ny, J.-F. (1989). Science Cognitive et Compréhension du Langage. Paris : Presses Universitaires de France.

Le Ny, J.F. (2005). Comment l' espriproduit du sens. Paris : Odile Jacob

Malt, B.C. (1989). An one-line investigation of prototype and exemplar strategies in classification. Journal of Experimental Psychology: Learning, Memory and Cognition, 15(4), 539-555.

Malt, B.C., \& Johnson, E.C. (1992). Do artifact concepts have cores? Journal of Memory and Language, 31, 195-217.

Malt, B.C., Sloman, S.A., \& Genneri, S. (2003). Universality and language specificity in object naming. Journal of Memory and Language, 49, 20-42.

Malt, B.C., Sloman, S.A., Genneri, S., Shi, M., \& Wang, Y. (1999). Knowing versus naming: Similarity and the linguistic categorization of artifacts. Journal of Memory and Language, 40, 230-262. 
Mandler, J. M. (1979). Categorical and schematic organization in memory. In C. R. Puff (Ed.), Memory organization and structure (pp. 259-302). New York: Academic.

Mandler, J. M. (1983). Representation. In J. H. Flavell \& E. M. Markman (Eds.), Cognitive development: Vol. 3. Handbook of child psychology (pp. 420-494). New York: Wiley.

Markman, A.B., \& Gentner, D. (1993a). Structural alignment during similarity comparisons. Cognitive Psychology, 25, 431-467.

Markman, A.B., \& Gentner, D. (1993b). Splitting the differences: A structural alignment view of similarity. Journal of Memory and Language, 32, 517-535.

Marques, J.F. (2002). An attribute is worth more than a category: Testing different semantic memory organization hypotheses in relation to the living/nonliving things dissociation. Cognitive Neuropsychology, 19, 463-478.

McCauley, R. N. (1987). The role of theories in a theory of concepts. In U. Neisser (Ed.), Concepts and conceptual development: Ecological and intellectual factors in categorization (pp. 288-309). Cambridge, MA: Cambridge University Press.

McClelland, J.L., \& Rumelhart, D.E. (1985). Distributed memory and the representation of general and specific information. Journal of Experimental Psychology: General, 114(2), 159-188.

McRae, K., \& Cree, G.S. (2002). Factors underlying category-specific semantic impairments. In E.M.E. Forde \& G.W. Humpreys, Category specificity in brain and mind (pp. 211-250). New York, Psychology Press. 
McRae, K., de Sa, V.R., \& Seidenberg, M.S. (1997). On the nature and scope of featural representations of word meaning. Journal of Experimental Psychology: General, 126(2), 99-130.

Medin, D.L., Altom, M.W., \& Murphy, T.D. (1984). Given versus induced category representations : use of prototype and exemplar information in classification. Journal of Experimental Psychology: Learning, Memory and Cognition, 10(3). 333-352.

Medin, D.L., \& Coley, J.D. (1998). Concepts and categorization. In J. Hochberg \& J.E. Cutting (Eds.), Handbook of perception and cognition at century' s end: History, philosophy, theory (pp. 403-439). New York: Academic Press.

Medin, D.L., Dewey, G.I., \& Murphy, T.D. (1983). Relationship between item and category learning : evidence that abstraction is not automatic. Journal of Experimental Psychology: Learning, Memory and Cognition, 9(4), 607-625.

Medin, D.L., \& Heit, E. (1998). Categorization. In D. Rumelhart \& J.E. Cutting (Eds.), Handbook of cognition and perception (pp. 99-143). San Diego: Academic Press.

Medin, D.L., Lynch, E.B., \& Solomon, K.O. (2000). Are there kinds of concepts? Annual Review of Psychology, 51, 121-147.

Medin, D.L., \& Ortony, A. (1989). Psychological essentialism. In S. Vosniadou \& A. Ortony (Eds.), Similarity and analogical reasoning (pp. 179-195). Cambridge, MA: Cambridge University Press.

Medin, D.L., \& Schaffer, M.M. (1978). Context theory of classification learning. Psychological Review, 85(3), 207-238. 
Medin, D.L., \& Shoben, E.J. (1988). Context and structure in conceptual combination. Cognitive Psychology, 20, 158-190.

Medin, D.L., \& Wattenmaker, W.D. (1987). Category cohesiveness, theories, and cognitive archaeology. In U. Neisser (Ed.), Concepts and conceptual development: Ecological and intellectual factors in categorization (pp. 25-62). Cambridge, MA: Cambridge University Press.

Medin, D.L., Wattenmaker, W.D., \& Hampton, S.E. (1987). Family resemblance, conceptual cohesiveness and category construction. Cognitive Psychology, 19, 242-279.

Murphy, G.L., \& Allopenna, P.D. (1994). The locus of knowledge effects in concept learning. Journal of Experimental Psychology: Learning, Memory and Cognition, 20, 904-919.

Murphy, G.L., \& Medin, D.L. (1985). The role of theories in conceptual coherence. Psychological Review, 92, 289-316.

Neisser, U. (1987). From direct perception to conceptual structure. In U. Neisser (Ed.), Concepts and conceptual development: Ecological and intellectual factors in categorization (pp. 11-24). Cambridge, MA: Cambridge University Press.

Neisser, U. (1988). What is ordinary memory the memory of? In U. Neisser \& E. Winograd (Eds.), Remembering reconsidered: Ecological and traditional approaches to the study of memory (pp. 356-373). Emory Symposia in Cognition, Cambridge, MA: Cambridge University Press.

Nelson, K. (1977). The syntagmatic-paradigmatic shift revisited: A review of research and theory. Psychological Bulletin, 84, 93-116. 
Nelson, K. (1996). Language in Cognitive Development: The Emergence of the Mediated Mind. New York: Cambridge University Press.

Nosofsky, R.M., Clark, S.E., \& Shin, H.J. (1989). Rules and exemplars in categorization, identification and recognition. Journal of Experimental Psychology: Learning, Memory and Cognition, 15(2), 282-304.

Nosofsky, R.M., Kruschke, J., \& McKinley, S. (1992). Combining exemplar-based category representations and connectionist learning rules. Journal of Experimental Psychology, Learning, Memory and Cognition, 18(2), 211-233.

Palmer, S.E., Rosch, E., \& Chase, P. (1981). Canonical perspective and the perception of objects. In J. Long \& A. Baddeley (Eds), Attention and Peformance (pp. 135151). Lawrence Erlbaum Associates, Hillsdale, New Jersey.

Pazzani, M. (1991). Influence of prior knowledge on concept acquisition: Experimental and computational results. Journal of Experimental Psychology: Learning, Memory and Cognition, 17(3), 416-432.

Pothos, E.M. (in press). The rules versus similarity distinction. Behavioral and Brain Sciences.

Pothos, E.M., \& Hahn, U. (2000). So concepts aren' t definitions, but do they have necessary or sufficient features? British Journal of Psychology, 91, 439-450.

Putnam, H. (1975). Mind, Language and Reality. New York: Cambridge University Press.

Reed, S.K. (1996). Cognition, Theory and Applications. International Thomson Publishing Inc. 
Rehder, B. (2003). A causal-model theory of conceptual representation and categorization. Journal of Experimental Psychology : Learning, Memory and Cognition, 29(6), 1141-1159.

Rehder, B., \& Hastie, R. (2001). Causal knowledge and categories: The effects of causal beliefs on categorization, induction, and similarity. Journal of Experimental Psychology: General, 130, 323-360.

Rehder, B., \& Hastie, R. (2004). Category coherence and category-based property induction. Cognition, 91, 113-153.

Rips, L.J. (1989). Similarity, typicality and categorization. In S. Vosniadou \& A. Ortony (Eds.), Similarity and analogical reasoning (pp. 21-59). Cambridge, MA: Cambridge University Press.

Rothschild, L., \& Haslam, N. (2003). Thirsty for H2O? Multiple essences and psychological essentialism. New Ideas in Psychology, 21, 31-41.

Rumelhart, D.E., \& Norman, D.A. (1981). Analogical processes in learning. In R.C. Anderson, Cognitive skills and their acquisition. Hillsdale, NJ: Erlbaum.

Schank, R.C., \& Abelson, R.P. (1977). Scripts, Plans, Goals, and Understanding. Hillsdale, NJ: Erlbaum.

Silveri, M.C., \& Gainotti, G. (1988). Interaction between vision and language in category-specific semantic impairment. Cognitive Neuropsychology, 5(6), 677709.

Sloman, S.A., \& Malt, B.C. (2003). Artifacts are not ascribed essences, nor are they treated as belonging to kinds. Language and Cognitive Processes, 18, 562-582. 
Sloutsky, V.M., \& Lo, Y.-F. (1999). How much does a shared name make things

similar? Part 1. Linguistic labels and the development of similarity judgment. Developmental Psychology, 35(6), 1478-1492.

Sloutsky, V.M., Lo, Y.-F., \& Fisher, A.V. (2001). How much does a shared name make things similar? Linguistic labels, similarity, and the development of inductive inference. Child Development, 72(6), 1695-1709.

Smith, E.E., Langston, C., \& Wisbett, R.E. (1992). The case for rules in reasoning. Cognitive Science, 16, 1-40.

Smith, E.E., Patanalo, A.L., \& Jonides, J. (1998). Alternative strategies of categorization. Cognition, 65, 167-196.

Smith, L. (1989). A model of perceptual classification in children and adults. Psychological Review, 96, 125-144.

Solomon, K.O., Medin, D.L., \& Lynch E.B. (1999). Concepts do more than categorize. Trends in Cognitive Sciences, 3, 99-105.

Strevens, M. (2000). The essentialist aspect of naive theories. Cognition, 74, 149-175.

Strevens, M. (2001). Only causation matters: reply to Ahn et al. Cognition, 82, 71-76.

Thibaut, J.P., Dupont, M., \& Anselme, P. (2002). Dissociations between categorization and similarity judgments as a result of learning feature distributions. Memory \& Cognition, 30(4), 647-656.

Tiberghien, A. (2003). Des connaissances naïves au savoir scientifique. In M. Kail \& M. Fayol, Les sciences cognitives et l' écolepp.353-413). Paris, Presses Universitaires de France. 
Tiberghien, G. (1999). La psychologie cognitive survivra-t-elle aux sciences cognitives? Psychologie Française, 44(3), 265-283.

Tijus, C., \& Cordier, F. (2003). Psychologie de la connaissance des objets, catégories et propriétés, tâches et domaines d' investigationL' Année Psychologique, 103223256.

Tijus, C., Poitrenaud, S., \& Barcenilla, J. (1997). Semantic networks of action for conception and evaluation of interfaces. Revue Internationale de Systémique, 11, 95-107.

Tyler, L.K., Moss, H.E., Durrant-Peatfield, M.R., \& Levy, J.P. (2000). Conceptual structure and the structure of concepts: A distributed account of category-specific deficits. Brain and Language, 75, 195-231.

Tulving, E. (1972). Episodic and semantic memory. In E. Tulving \& W. Donaldson (Eds.), Organization of memory (pp. 382-403). New York: Academic Press.

Tulving, E. (1983). Elements of Episodic Memory. Oxford: Oxford University Press.

Warrington, E.K., \& Shallice, T. (1984). Category-specific impairments. Brain, $107,829-852$. 
Notes des Auteurs.

Soulaimane Chemlal et Françoise Cordier, LaCo, FRE CNRS 2725, Université de Poitiers (France).

Nous remercions Serge Larochelle (Université de Montréal), Jean-François Le Ny (Université Paris-sud) et deux experts anonymes pour leurs commentaires et suggestions concernant une version antérieure de ce texte.

La correspondance concernant cet article sera adressée à Françoise Cordier, 99, avenue du Recteur Pineau, 86000 Poitiers. France.

Courriel : francoise.cordier@mshs.univ-poitiers.fr 